\title{
Nilpotency of the Ordinary Lie-algebra of an $n$-Lie Algebra
}

\author{
Côme Chancel Likouka ${ }^{1}$
}

\author{
${ }^{1}$ Université Marien Ngouabi, BP: 69, Brazzaville, Congo
}

Correspondence: Côme Chancel Likouka, Université Marien Ngouabi, BP: 69, Brazzaville, Congo. E-mail: clikouka@gmail.com

\author{
Received: November 11, 2016 Accepted: February 3, 2017 Online Published: March 16, 2017 \\ doi:10.5539/jmr.v9n2p79 \\ URL: https://doi.org/10.5539/jmr.v9n2p79
}

The author was supported by the International Mathematical Union under the project IMU-Congo.

\begin{abstract}
In this paper, we generalize to $n$-Lie algebras a corollary of the well-known Engel's theorem which offers some justification for the terminology "nilpotent" and we construct a nilpotent ordinary Lie algebra from a nilpotent $n$-Lie algebra.
\end{abstract}

Keywords: Lie algebra, $n$-Lie algebra, nilpotency

\section{Introduction}

(Filipov, 1985) Introduced a generalization of a Lie algebra, which he called an $n$-Lie algebra. The Lie product is taken between $n$ elements of the algebra instead of two. This new bracket is $n$-linear, anti-symmetric and satisfies a generalization of the Jacobi identity.

(Bossoto,Okassa, \& Omporo, 2013) Associate to an $n$-Lie algebra, a Lie algebra called the ordinary Lie algebra.

In this paper, we generalize to $n$-Lie algebras a corollary of the well-known Engel's theorem and we construct a nilpotent ordinary Lie algebra from a nilpotent $n$-Lie algebra.

\section{1 -Lie Algebra Structure}

In the following, $K$ will denote a commutative field with characteristic zero.

An $n$-Lie algebra $\mathcal{G}$ over $K$ is a vector space together with a multilinear fully skewsymmetric map

$$
\{, \ldots,\}: \mathcal{G}^{n}=\mathcal{G} \times \mathcal{G} \times \ldots \times \mathcal{G} \longrightarrow \mathcal{G},\left(x_{1}, x_{2}, \ldots, x_{n}\right) \longmapsto\left\{x_{1}, x_{2}, \ldots, x_{n}\right\},
$$

such that

$$
\left\{x_{1}, x_{2}, \ldots, x_{n-1},\left\{y_{1}, y_{2}, \ldots, y_{n}\right\}\right\}=\sum_{i=1}^{n}\left\{y_{1}, y_{2}, \ldots, y_{i-1},\left\{x_{1}, x_{2}, \ldots, x_{n-1}, y_{i}\right\}, y_{i+1}, \ldots, y_{n}\right\}
$$

for all $x_{1}, x_{2}, \ldots, x_{n-1}, y_{1}, y_{2}, \ldots, y_{n}$ elements of $\mathcal{G}$.

The above equation is called the generalized Jacobi Identity.

A subspace $\mathcal{G}_{0}$ of $\mathcal{G}$ is called an $n$-Lie subalgebra if for any $y_{1}, y_{2}, \ldots, y_{n} \in \mathcal{G}_{0},\left\{y_{1}, y_{2}, \ldots, y_{n}\right\} \in \mathcal{G}_{0}$.

Let $\mathcal{G}_{1}, \mathcal{G}_{2}, \ldots, \mathcal{G}_{n}$ be subalgebras of n-Lie algebra $\mathcal{G}$ and let $\left\{\mathcal{G}_{1}, \mathcal{G}_{2}, \ldots, \mathcal{G}_{n}\right\}$ denote the subspace of $\mathcal{G}$ generated by all vectors $\left\{x_{1}, x_{2}, \ldots, x_{n}\right\}$, where $x_{i} \in \mathcal{G}_{i}$ for $i=1,2, \ldots, n$. The subalgebra $\{\mathcal{G}, \mathcal{G}, \ldots, \mathcal{G}\}$ is called the derived algebra of $\mathcal{G}$, and is denoted by $\mathcal{G}^{1}$. If $\mathcal{G}^{1}=0$, then $\mathcal{G}$ is called an abelian n-Lie algebra.

Using the derivation $a d\left(x_{1}, x_{2}, \ldots, x_{n-1}\right): \mathcal{G} \longrightarrow \mathcal{G}, y \longmapsto\left\{x_{1}, x_{2}, \ldots, x_{n-1}, y\right\}$, we can rephrase this definition as follows:

A vector subspace $\mathcal{G}_{0}$ of $\mathcal{G}$ is an $n$-Lie subalgebra of $\mathcal{G}$ if $a d\left(x_{1}, x_{2}, \ldots, x_{n-1}\right)\left(\mathcal{G}_{0}\right) \subset \mathcal{G}_{0}$ for any $x_{1}, x_{2}, \ldots, x_{n-1} \in \mathcal{G}_{0}$. That is, $\operatorname{ad}\left(\mathcal{G}_{0}, \mathcal{G}_{0}, \ldots, \mathcal{G}_{0}\right)\left(\mathcal{G}_{0}\right) \subset \mathcal{G}_{0}$.

A subspace $\mathcal{I}$ of $\mathcal{G}$ is called an ideal if $\left\{x, y_{1}, y_{2}, \ldots, y_{n-1}\right\} \in \mathcal{I}$ for any $x \in \mathcal{I}$, and for any $y_{1}, y_{2}, \ldots, y_{n-1} \in \mathcal{G}$. That is equivalent to say that $\operatorname{ad}(\mathcal{G}, \ldots, \mathcal{G})(\mathcal{I}) \subset \mathcal{I}$.

\subsection{The Ordinary Lie Algebra of an n-Lie Algebra}

Let $\mathcal{G}$ be an $n$-Lie algebra over a field $K$. (Bossoto et al., 2013) associate to $\mathcal{G}$ a Lie algebra called the ordinary Lie algebra. This construction goes as presented below:

Consider the map

$$
\mathcal{G}^{n-1} \longrightarrow \operatorname{Der}_{K}(\mathcal{G}),\left(x_{1}, x_{2}, \ldots, x_{n-1}\right) \longmapsto \operatorname{ad}\left(x_{1}, x_{2}, \ldots, x_{n-1}\right)
$$


where $\operatorname{Der}_{K}(\mathcal{G})$ denote the set of $K$-derivations of $\mathcal{G}$.

Denote by $\Lambda_{K}^{n-1}(\mathcal{G})$, the $(n-1)$-exterior power of the $K$-vector space $\mathcal{G}$, there exists a unique $K$-linear map

$$
a d_{\mathcal{G}}: \Lambda_{K}^{n-1}(\mathcal{G}) \longrightarrow \operatorname{Der}_{K}(\mathcal{G})
$$

such that

$$
\operatorname{ad}_{\mathcal{G}}\left(x_{1} \Lambda x_{2} \Lambda \ldots \Lambda x_{n-1}\right)=\operatorname{ad}\left(x_{1}, x_{2}, \ldots, x_{n-1}\right)
$$

for all $x_{1}, x_{2}, \ldots, x_{n-1} \in \mathcal{G}$.

When $f: W \longrightarrow W$ is an endomorphism of a $K$-vector space $W$ and when $\Lambda_{K}(W)$ is the $K$-exterior algebra of $W$, then there exists a unique derivation of degree zero

$$
D_{f}: \Lambda_{K}(W) \longrightarrow \Lambda_{K}(W)
$$

such that, for $p \in \mathbb{N}$,

$$
D_{f}\left(w_{1} \Lambda w_{2} \Lambda \ldots \Lambda w_{p}\right)=\sum_{i=1}^{p} w_{1} \Lambda w_{2} \Lambda \ldots \Lambda w_{i-1} \Lambda f\left(w_{i}\right) \Lambda w_{i+1} \Lambda \ldots \Lambda w_{p}
$$

for all $w_{1}, w_{2}, \ldots, w_{p}$ elements of $W$.

Proposition 1 For all $s_{1}$ and $s_{2}$ elements of $\Lambda_{K}^{n-1}(\mathcal{G})$, then we have simultaneously

$$
\left[a d_{\mathcal{G}}\left(s_{1}\right), a d_{\mathcal{G}}\left(s_{2}\right)\right]=a d_{\mathcal{G}}\left(D_{a d_{\mathcal{G}}\left(s_{1}\right)}\left(s_{2}\right)\right)
$$

and

$$
\left[a d_{\mathcal{G}}\left(s_{1}\right), a d_{\mathcal{G}}\left(s_{2}\right)\right]=a d_{\mathcal{G}}\left(-D_{a d_{\mathcal{G}}\left(s_{2}\right)}\left(s_{1}\right)\right)
$$

where [, ] denotes the usual bracket of endomorphisms.

We denote by $\mathcal{V}_{K}(\mathcal{G})$ the $K$-subspace of $\Lambda_{K}^{n-1}(\mathcal{G})$ generated by the elements of the form $D_{a d_{\mathcal{G}}\left(s_{1}\right)}\left(s_{2}\right)+D_{a d_{\mathcal{G}}\left(s_{2}\right)}\left(s_{1}\right)$ where $s_{1}$ and $s_{2}$ describe $\Lambda_{K}^{n-1}(\mathcal{G})$.

Let

$$
\Lambda_{K}^{n-1}(\mathcal{G}) \longrightarrow \Lambda_{K}^{n-1}(\mathcal{G}) / \mathcal{V}_{K}(\mathcal{G}), s \longmapsto \bar{s},
$$

be the canonical surjection. Given the foregoing, we conclude that $a d_{\mathcal{G}}\left[\mathcal{V}_{K}(\mathcal{G})\right]=0$.

We denote by

$$
\widetilde{\operatorname{ad}_{\mathcal{G}}}: \Lambda_{K}^{n-1}(\mathcal{G}) / \mathcal{V}(\mathcal{G}) \longrightarrow \operatorname{Der}_{K}(\mathcal{G})
$$

the unique linear map such that

$$
\widetilde{a d_{\mathcal{G}}}(\bar{s})=a d_{\mathcal{G}}(s)
$$

for all $s \in \Lambda_{K}^{n-1}(\mathcal{G})$.

Theorem 2 When $(\mathcal{G},\{, \ldots\}$,$) is a n$-Lie algebra, then the map

$$
[,]:\left[\Lambda_{K}^{n-1}(\mathcal{G}) / \mathcal{V}_{K}(\mathcal{G})\right]^{2} \longrightarrow \Lambda_{K}^{n-1}(\mathcal{G}) / \mathcal{V}_{K}(\mathcal{G}),\left(\overline{s_{1}}, \overline{s_{2}}\right) \longmapsto \overline{D_{a d_{\mathcal{G}}\left(s_{1}\right)}\left(s_{2}\right)},
$$

depends only on $\overline{s_{1}}$ and $\overline{s_{2}}$, and defines an ordinary Lie algebra structure on $\Lambda_{K}^{n-1}(\mathcal{G}) / \mathcal{V}_{K}(\mathcal{G})$.

Proposition 3 If a subspace $\mathcal{G}_{0}$ of an n-Lie algebra $\mathcal{G}$ is stable for the representation

$$
\widetilde{a d_{\mathcal{G}}}: \Lambda_{K}^{n-1}(\mathcal{G}) / \mathcal{V}_{K}(\mathcal{G}) \longrightarrow \operatorname{Der}_{K}(\mathcal{G}), \bar{s} \longmapsto a d_{\mathcal{G}}(s),
$$

then $\mathcal{G}_{0}$ is an ideal of the $n$-Lie algebra $\mathcal{G}$. 


\section{Nilpotency of the Ordinary Lie Algebra}

An $n$-Lie algebra $\mathcal{G}$ is nilpotent if $\mathcal{G}$ satisfies $\mathcal{G}^{r}=0$ for some $r \geq 0$, where $\mathcal{G}^{0}=\mathcal{G}$ and $\mathcal{G}^{r}$ is defined by induction, $\mathcal{G}^{r+1}=\left[\mathcal{G}^{r}, \mathcal{G}, \mathcal{G}, \cdots, \mathcal{G}\right]$ for $r \geq 0$.

Proposition 4 Let $\mathcal{G}$ be an $n$-Lie algebra over a field $K$. If $\mathcal{G} \neq 0$ is nilpotent then $\mathcal{Z}(\mathcal{G}) \neq 0$.

Proof. Let us suppose $\mathcal{Z}(\mathcal{G})=0$.

Nilpotency of $\mathcal{G}$ implies that there exists an integer $k \geq 0$ such that $\mathcal{G}^{k-1} \neq 0$ and $\mathcal{G}^{k}=0$.

$$
\begin{aligned}
0=\mathcal{G}^{k} & =\left\{\mathcal{G}^{k-1}, \mathcal{G}, \mathcal{G}, \ldots, \mathcal{G}\right\} \\
& =\left\{\mathcal{G}, \mathcal{G}, \ldots, \mathcal{G}, \mathcal{G}^{k-1}\right\} \\
& =\operatorname{ad}(\mathcal{G}, \mathcal{G}, \ldots, \mathcal{G})\left(\mathcal{G}^{k-1}\right) \\
& =0
\end{aligned}
$$

Then $\mathcal{G}^{k-1} \subset \mathcal{Z}(\mathcal{G})$.

Therefore $0 \neq \mathcal{G}^{k-1} \subset \mathcal{Z}(\mathcal{G})=0$ which is impossible.

Thus $\mathcal{Z}(\mathcal{G}) \neq 0$.

Below we give the statement of the Engel's theorem and its corollary for Lie algebras:

Theorem 5 (Engel) Let $\rho: \mathcal{G} \rightarrow \operatorname{End}(V)$ be a linear representation of $\mathcal{G}$ on the vector space $V$ such that $\rho(x)$ is nilpotent for each $x \in \mathcal{G}$. If $V \neq(0)$, then there

exists $v \in V, v \neq 0$ such that $\rho(x) v=0$ for all $x \in \mathcal{G}$.

Corollary $6 \mathcal{G}$ is nilpotent if and only if $a d x$ is nilpotent for each $x \in \mathcal{G}$.

Now we're going to give a generalization to $n$-Lie algebras of the above corollary:

Theorem 7 Let $\mathcal{G}$ be an $n$-Lie algebra over a field $K . \mathcal{G}$ is nilpotent if and only if $a d\left(x_{1}, x_{2}, \ldots, x_{n-1}\right)$ is nilpotent for any $x_{1}, x_{2}, \ldots, x_{n-1} \in \mathcal{G}$.

To prove the Theorem, one needs some Lemmas:

Lemma 8 Let $\mathcal{G}$ be an $n$-Lie algebra, $\mathcal{Z}(\mathcal{G})$ the center of $\mathcal{G}$ and $\pi: \mathcal{G} \rightarrow \mathcal{G} / \mathcal{Z}(\mathcal{G})$ the canonical surjection. For any $x_{1}, x_{2}, \ldots, x_{n-1} \in \mathcal{G}$, if $\operatorname{ad}\left(x_{1}, x_{2}, \ldots, x_{n-1}\right): \mathcal{G} \rightarrow \mathcal{G}$ is nilpotent, then the unique linear map

$$
\overline{a d_{\mathcal{G}}\left(x_{1}, x_{2}, \ldots, x_{n-1}\right)}: \mathcal{G} / \mathcal{Z}(\mathcal{G}) \rightarrow \mathcal{G} / \mathcal{Z}(\mathcal{G}), \bar{y} \mapsto \overline{\left\{x_{1}, x_{2}, \ldots, x_{n-1}, y\right\}}
$$

such that $\pi \circ \overline{a d_{\mathcal{G}}\left(x_{1}, x_{2}, \ldots, x_{n-1}\right)}=a d_{\mathcal{G}}\left(x_{1}, x_{2}, \ldots, x_{n-1}\right) \circ \pi$ is nilpotent.

Proof. It's clear that $a d\left(x_{1}, x_{2}, \ldots, x_{n-1}\right)[\mathcal{Z}(\mathcal{G})]=0$. We denote by

$$
\overline{a d_{\mathcal{G}}\left(x_{1}, x_{2}, \ldots, x_{n-1}\right)}: \mathcal{G} / \mathcal{Z}(\mathcal{G}) \rightarrow \mathcal{G} / \mathcal{Z}(\mathcal{G}), \bar{y} \mapsto \overline{\left\{x_{1}, x_{2}, \ldots, x_{n-1}, y\right\}}
$$

the unique linear map such that $\pi \circ \overline{a d_{\mathcal{G}}\left(x_{1}, x_{2}, \ldots, x_{n-1}\right)}=a d_{\mathcal{G}}\left(x_{1}, x_{2}, \ldots, x_{n-1}\right) \circ \pi$.

$a d\left(x_{1}, x_{2}, \ldots, x_{n-1}\right)$ nilpotent, then there exists $k \geq 0$ such that $\left(a d_{\mathcal{G}}\left(x_{1}, x_{2}, \ldots, x_{n-1}\right)\right)^{k}=0$. We have: $\left(\overline{a d_{\mathcal{G}} h}\right)^{k} \circ \pi=$ $\pi \circ\left(a d_{\mathcal{G}} h\right)^{k}=0$. Since $\pi$ is surjective $\Rightarrow\left(\overline{a d_{\mathcal{G}}\left(x_{1}, x_{2}, \ldots, x_{n-1}\right)}\right)^{k}=0$ ie $\overline{a d_{\mathcal{G}}\left(x_{1}, x_{2}, \ldots, x_{n-1}\right)}$ is nilpotent.

Lemma 9 If for any $x_{1}, x_{2}, \ldots, x_{n-1} \in \mathcal{G}, a d\left(x_{1}, x_{2}, \ldots, x_{n-1}\right): \mathcal{G} \rightarrow \mathcal{G}$ is nilpotent, then $\mathcal{Z}(\mathcal{G}) \neq(0)$.

Proof. Using the well-known Engel's theorem, there exists $u \in \mathcal{G}, u \neq 0$, such that $\operatorname{ad}\left(x_{1}, x_{2}, \ldots, x_{n-1}\right)(u)=0$, for any $x_{1}, x_{2}, \ldots, x_{n-1} \in \mathcal{G}$. That implies $u \in \mathcal{Z}(\mathcal{G})$. And as $u \neq 0$, thus $\mathcal{Z}(\mathcal{G}) \neq(0)$. We are done.

The set $\left\{a d\left(x_{1}, x_{2}, \ldots, x_{n-1}\right) / a d\left(x_{1}, x_{2}, \ldots, x_{n-1}\right)\right.$ is nilpotent for any $\left.x_{1}, x_{2}, \ldots, x_{n-1} \in \mathcal{G}\right\}$ is a Lie subalgebra of $\operatorname{End}_{\mathbb{k}}(\mathcal{G})$.

Proof. " $\Rightarrow "$ :

$\mathcal{G}$ nilpotent implies that there exists $k \geq 0$ such that $\mathcal{G}^{k-1} \neq 0$ and $\mathcal{G}^{k}=0$. 


$$
\begin{aligned}
0 & =\mathcal{G}^{k}=\left\{\mathcal{G}, \mathcal{G}, \ldots, \mathcal{G}, \mathcal{G}^{k-1}\right\} \\
& =\operatorname{ad}(\mathcal{G}, \mathcal{G}, \ldots, \mathcal{G})\left(\mathcal{G}^{k-1}\right) \\
& =\operatorname{ad}(\mathcal{G}, \mathcal{G}, \ldots, \mathcal{G})\left\{\mathcal{G}, \mathcal{G}, \ldots, \mathcal{G}, \mathcal{G}^{k-2}\right\} \\
& =\operatorname{ad}(\mathcal{G}, \mathcal{G}, \ldots, \mathcal{G})\left[\operatorname{ad}(\mathcal{G}, \mathcal{G}, \ldots, \mathcal{G})\left(\mathcal{G}^{k-2}\right)\right] \\
& =\underbrace{[a d(\mathcal{G}, \mathcal{G}, \ldots, \mathcal{G}) \circ \operatorname{ad}(\mathcal{G}, \mathcal{G}, \ldots, \mathcal{G}) \circ \operatorname{ad}(\mathcal{G}, \mathcal{G}, \ldots, \mathcal{G}) \circ \ldots \circ \operatorname{ad}(\mathcal{G}, \mathcal{G}, \ldots, \mathcal{G})](\mathcal{G})}_{k \text {-times }} \\
& =[a d(\mathcal{G}, \mathcal{G}, \ldots, \mathcal{G})]^{k}(\mathcal{G})
\end{aligned}
$$

i.e $\left[\operatorname{ad}\left(x_{1}, x_{2}, \ldots, x_{n-1}\right)\right]^{k}=0$ for any $x_{1}, x_{2}, \ldots, x_{n-1} \in \mathcal{G}$.

Thus $\operatorname{ad}\left(x_{1}, x_{2}, \ldots, x_{n-1}\right)$ is nilpotent.

$" \Longleftarrow "$ we prove by induction on the dimension of $\mathcal{G}$.

- $\operatorname{dim} \mathcal{G}=1, \operatorname{ad}\left(x_{1}, x_{2}, \ldots, x_{n-1}\right): \mathcal{G} \rightarrow \mathcal{G}$ is nilpotent $\Rightarrow \operatorname{ad}\left(x_{1}, x_{2}, \ldots, x_{n-1}\right)(y)=0$ for any $x_{1}, x_{2}, \ldots, x_{n-1}, y \in \mathcal{G}$, that is $\mathcal{G}$ is commutative. Thus $\operatorname{ad}\left(\mathcal{G}^{n-1}\right)(\mathcal{G})=0$ ie $\mathcal{G}^{1}=0$. Therefore $\mathcal{G}$ is nilpotent.

- Suppose the assumption true for $\operatorname{dim} \mathcal{G}=n$.Let's verify the assumption for $\operatorname{dim} \mathcal{G}=n+1$.

$\operatorname{ad}\left(x_{1}, x_{2}, \ldots, x_{n-1}\right)$ nilpotent for any $x_{1}, x_{2}, \ldots, x_{n-1} \in \mathcal{G}$, then from Lemma $8, \overline{a d_{\mathcal{G}}\left(x_{1}, x_{2}, \ldots, x_{n-1}\right)}: \mathcal{G} / \mathcal{Z}(\mathcal{G}) \rightarrow \mathcal{G} / \mathcal{Z}(\mathcal{G})$ is nilpotent for any $x_{1}, x_{2}, \ldots, x_{n-1} \in \mathcal{G} . \Rightarrow \mathcal{G} / \mathcal{Z}(\mathcal{G})$ is nilpotent and $\mathcal{Z}(\mathcal{G}) \neq 0$ from Lemma 9 . $\mathcal{G} / \mathcal{Z}(\mathcal{G})$ nilpotent, there exists $k \geq 0$ such that $[\mathcal{G} / \mathcal{Z}(\mathcal{G})]^{k}=0$. As $\pi: \mathcal{G} \rightarrow \mathcal{G} / \mathcal{Z}(\mathcal{G})$, then $[\mathcal{G} / \mathcal{Z}(\mathcal{G})]^{k}=\pi\left(\mathcal{G}^{k}\right)=0$ since $\pi$ is surjective. Thus $\mathcal{G}^{k} \subset \mathcal{Z}(\mathcal{G}) . \mathcal{G}^{k+1}=\operatorname{ad}\left(\mathcal{G}^{n-1}\right)\left(\mathcal{G}^{k}\right) \subset \operatorname{ad}\left(\mathcal{G}^{n-1}\right)(\mathcal{Z}(\mathcal{G}))=0$. Therefore $\mathcal{G}$ is nilpotent. That ends the proof.

Below we give the statement of the main theorem we obtained:

Theorem 10 If $\mathcal{G}$ is a nilpotent n-Lie algebra over a field $\mathbb{k}$ and if $\widetilde{a d_{\mathcal{G}}}: \Lambda_{K}^{n-1}(\mathcal{G}) / \mathcal{V}_{K}(\mathcal{G}) \longrightarrow \operatorname{Der}_{K}(\mathcal{G}), \bar{s} \longmapsto a d_{\mathcal{G}}(s)$,is the canonical representation of $\Lambda_{K}^{n-1}(\mathcal{G}) / \mathcal{V}_{K}(\mathcal{G})$ in $\mathcal{G}$, then $\left[\Lambda_{K}^{n-1}(\mathcal{G}) / \mathcal{V}_{K}(\mathcal{G})\right] / \operatorname{Kerad}_{\mathcal{G}}$ is a nilpotent Lie algebra.

Proof. Let $\mathcal{G}$ be an n-Lie algebra. Then the mapping

$$
\mathcal{G}^{n-1} \longrightarrow \operatorname{Der}_{K}(\mathcal{G}),\left(x_{1}, x_{2}, \ldots, x_{n-1}\right) \longmapsto a d\left(x_{1}, x_{2}, \ldots, x_{n-1}\right),
$$

induces a representation $\widetilde{a d_{\mathcal{G}}}: \Lambda_{K}^{n-1}(\mathcal{G}) / \mathcal{V}_{K}(\mathcal{G}) \longrightarrow \operatorname{Der}_{K}(\mathcal{G}), \bar{s} \longmapsto a d_{\mathcal{G}}(s)$ of $\Lambda_{K}^{n-1}(\mathcal{G}) / \mathcal{V}_{K}(\mathcal{G})$ in $\mathcal{G}$. When $\mathcal{G}$ is a nilpotent n-Lie algebra then $\widetilde{a d_{\mathcal{G}}}\left(\Lambda_{K}^{n-1}(\mathcal{G}) / \mathcal{V}_{K}(\mathcal{G})\right)$ is a Lie subalgebra of $\operatorname{Der}_{K}(\mathcal{G})$ whose all elements are nilpotent.Thus $\widetilde{\operatorname{ad}_{\mathcal{G}}}\left(\Lambda_{K}^{n-1}(\mathcal{G}) / \mathcal{V}_{K}(\mathcal{G})\right)$ is a nilpotent Lie algebra. Therefore $\left[\Lambda_{K}^{n-1}(\mathcal{G}) / \mathcal{V}_{K}(\mathcal{G})\right] / \operatorname{Kerad}_{\mathcal{G}}$ is a nilpotent Lie algebra.

\section{Acknowledgements}

We are very grateful to Professor Eugène Okassa for his advice and for his help.

\section{References}

Bossoto, B. G. R., Okassa, E., \& Omporo, M. (2013). Lie algebra of an n-Lie algebra.

Bourbaki, N. (1974). Elements of Mathematics: Algebra 1, chapters 1-3 . Hermann.

Filipov, V. T. (1985). n-Lie algebras. Sibirsk. Mat. Zh., 39(6), 126-140. (English translation: (1985). Siberian Math. J., 26(6), 879-891).

Filipov, V. T. (1998). On n-Lie algebra of jacobians. Sibirsk. Mat. Zh., 26(6), 126-140. (English translation: (1985). Siberian Math. J., 26(6), 879-891.)

Kasymov, S. M. (1987). Theory of n-Lie algebras. Algebra i Logika, 26(3), 277-297. (English translation: (1988). Algebra and Logic 26, 155-166.) https://doi.org/10.1007/BF02009328

Ling, W. X. (1993). On the structure of n-Lie algebras . Siegen, PhD thesis.

Pozhidaev, A. P. (1993). n-Lie algebras . Algebra and Logic, 38(3), 181-192. https://doi.org/10.1007/BF02671742

Rotkiewicz, M. L. (2005). Cohomology Ring of n-Lie Algebras. Extracta mathematicae, 20(3), 219-232.

Williams, M. P. (2009, June). Nilpotent n-Lie Algebras . Communications in Algebra, 37(6), 1843-1849. https://doi.org/10.1080/00927870802108007 


\section{Copyrights}

Copyright for this article is retained by the author(s), with first publication rights granted to the journal.

This is an open-access article distributed under the terms and conditions of the Creative Commons Attribution license (http://creativecommons.org/licenses/by/4.0/). 